\title{
Image registration method for assessing 3D hip alignment and implant position during standing posture
}

\author{
Koichi KOBAYASHI*, Shin KAI**, Makoto SAKAMOTO*, Yuji TANABE***, \\ Kunihiko TOKUNAGA****, Izumi MINATO*****, Takashi SATO****** and Yoshio KOGA******* \\ *School of Health Sciences, Faculty of Medicine, Niigata University, \\ 2-746 Asahimachi-dori, Chuo-ku, Niigata 951-8518, Japan \\ E-mail: kobayasi@clg.niigata-u.ac.jp \\ ${ }^{* *}$ Center for Fostering Innovative Leadership, Niigata University and Medacta Japan Co., Ltd., \\ 2-8050 Ikarashi, Nishi-ku, Niigata 950-2181, Japan and 2-17-8 Nagatacho, Chiyoda-ku, Tokyo 100-0014, Japan \\ ${ }^{* * *}$ Department of Mechanical and Production Engineering, Niigata University, \\ 2-8050 Ikarashi, Nishi-ku, Niigata 950-2181, Japan \\ ${ }^{* \star * *}$ Niigata Hip Joint Center, Kameda Daiichi Hospital, \\ 2-5-22 Nishimachi, Konan-ku, Niigata 950-0165, Japan \\ **** Department of Orthopaedic Surgery, Niigata Rinko Hospital, \\ 1-114-3 Momoyamacho, Higashi-ku, Niigata 950-0051, Japan \\ ******Department of Orthopaedic Surgery, Niigata Medical Center, \\ 3-27-11 Kobari, Nishi-ku, Niigata 950-2022, Japan \\ ******* Division of Orthopaedic Biomechanics, Hokuetsu Hospital and Niohji Onesen Hospital \\ 2-20-19 Midori-cho, Shibata 957-0018, Japan and 452 Toramaru, Shibata 957-0332, Japan
}

Received 30 July 2013

\begin{abstract}
The range of motion (ROM) resulting from total hip arthroplasty (THA) depends on the relative orientation of the femoral and acetabular components, and malpositioning of components is a possible cause of several complications. The purpose of this study was to validate a 3-dimensional (3D) lower extremity alignment assessment system (3D LAAS) for the measurement of alignment of natural and implanted hip joints and implant position with respect to the target bone with the subject in a standing position. Sawbone femur and pelvis and femoral and acetabular components of a total hip arthroplasty system were used. Three spherical markers were attached to each sawbone and each component to define the local coordinate system. Outlines of the 3D bone models and component computer-aided design models were projected onto extracted contours of the femur, pelvis, and implants in calibrated frontal and oblique X-ray images. The 3D position of each model was recovered by minimizing the differences between projected outlines and contours. Mean absolute errors were less than $1.27 \pm 0.79 \mathrm{~mm}$ and $0.99 \pm 0.52^{\circ}$ for femur and pelvis, $1.64 \pm 0.66 \mathrm{~mm}$ and $1.54 \pm 0.39^{\circ}$ for femoral and acetabular components, $1.58 \pm 0.29 \mathrm{~mm}$ and $1.75 \pm 0.69^{\circ}$ for femur and femoral component, and $1.51 \pm 0.49$ $\mathrm{mm}$ and $1.24 \pm 0.14^{\circ}$ for pelvis and acetabular component, indicating that the 3D LAAS is applicable to measure the alignment of natural and implanted hip joints and implant position after THA.
\end{abstract}

Key words : Biomechanics, Hip joint, Alignment, Total hip arthroplasty, Image registration

\section{Introduction}

The hip joint is a ball-and-socket joint with the femoral head as the ball and the acetabulum of the pelvic bone as the socket. A wide range of motion (ROM) at the hip enables flexion/extension, abduction/adduction, and internal/external rotation during various daily activities. Disorder of the hip with reduced ROM can thus considerably impede daily activities. Total hip arthroplasty (THA) is a surgical treatment to restore the biomechanical function of the diseased hip joint by replacing the femoral neck and head and the acetabulum with an artificial stem, head and cup. In Japan, about 70,000 patients underwent THA in 2011. The resulting ROM of the THA depends on the relative orientation of femoral 
and acetabular components (D'Lima et al., 2000, Yoshimine, 2006), and malpositioning of components is a possible cause of complications such as impingement (Malik, et al., 2007), edge loading (Mellon, et al., 2011), dislocation (Jolles, et al., 2002), implant loosening and wear (Kesteris, et al., 2003). Numerous studies have therefore been carried out to develop accurate and precise methods for pre-operative planning and intra-operative positioning of THA. These attempts have produced a computer navigation system (Jolles, et al, 2004) that utilizes computed tomography (CT) to visualize or reconstruct the 3-dimensional (3D) anatomical structure of the hip in place of two-dimensional (2D) radiographs. Although a computer navigation system offers adequate accuracy and precision for clinical usage (Iwana, et al., 2013, Kitada, et al., 2011), pre-operative component positioning is determined using CT data of the hip obtained with the patient in a supine position. Since the posterior tilt of the pelvis relative to the femur differs between lying and standing postures, considerable bias in the alignment between the implanted femur and pelvis may exist during standing. Also, the relative position of the component with respect to the target bone would be affected, resulting in malpositioning. These may lead to reduced ROM after THA and the above-mentioned complications. However, although several studies have been reported to evaluate hip joint kinematics (Komistek, et al., 2002, Koyanagi, et al., 2011, Penney, et al., 2007, Tsai, et al., 2013, Martin, et al., 2011, Lin, et al., 2013), 3D alignment of the natural or implanted hip joint in a standing posture has not been well quantified in relation to the entire lower extremity, as few practical methods have been reported to date.

We have previously developed a 3D lower extremity alignment assessment system (3D LAAS) with the subject in a standing position that has been applied to alignment assessment of normal subjects, pre-operative planning and assessment of post-operative alignment for total knee arthroplasty (TKA) (Sato, et al., 2004, Sato, et al., 2007, Kobayashi, et al., 2009, Ariumi, et al., 2010). However, the feasibility and validity of 3D LAAS for assessing alignment of the hip joint and implant position of THA in a standing position has not previously been determined. The purpose of this study was to adapt and validate the 3D LAAS for measuring the alignment of natural and implanted hip joints and implant position with respect to the target bone using in-vitro sawbone specimens.

\section{Materials and Methods}

\subsection{Model preparation}

Sawbone femur and pelvis and femoral and acetabular components of the Perfix 910 total hip system (Japan Medical Materials, Osaka, Japan) were used. Three spherical acrylic markers (diameter, $25 \mathrm{~mm}$ ) were attached to the femur and pelvis, while 3 spherical stainless markers (diameter, $5 \mathrm{~mm}$ ) were attached to the femoral and acetabular components. Computed tomography of the sawbone femur and pelvis with these markers was performed to allow the reconstruction of 3D surface models with the accuracy and precision of $0.40 \pm 0.33 \mathrm{~mm}$, determined by comparing the dimensions of the surface model with actual values (Sasagawa, et al., 2008). The local coordinate system of each surface model was defined based on the central coordinates of the markers attached to the surface model using a coordinate system creator (ModelViewer; LEXI, Tokyo, Japan). For the components, computer-aided design (CAD) models were provided by the manufacturer. Using the coordinate system creator (ModelViewer), spheres of $5 \mathrm{~mm}$ diameter were placed at the actual positions of the markers as measured by a 3D-coordinate measuring machine (BH504; Mitutoyo, Kawasaki, Japan). A local coordinate system for each CAD model was then defined based on the central coordinates of the sphere models (Fig. 1).

\subsection{Determination of relative position}

The sawbone femur and pelvis were rigidly fixed at extension using a custom holder. The 3D position for each sawbone was determined based on the central coordinates of the markers measured using the 3D-coordinate measuring machine (Fig. 2). After biplanar X-ray exposure (described later), the pelvis was tilted anteriorly about $15^{\circ}$ and fixed again. The 3D position for each bone was then measured using the 3D-coordinate measuring machine and biplanar Xray images were captured. This process was repeated once more for the pelvis tilted posteriorly about $15^{\circ}$. The femoral and acetabular components were installed by an orthopedic joint replacement surgeon according to the procedure suggested by the manufacturer. For the implanted femur and pelvis, both the 3D position measurements and X-ray exposure as above were repeated three times in the three positions (extension, anterior tilt and posterior tilt of the pelvis). Relative positions were obtained in full 6 degrees of freedom (6-DOF) parameters for the femur with respect to the pelvis, the femoral component to the acetabular component, the femoral component to the femur, and the acetabular component to the pelvis, from the corresponding pairs of $3 \mathrm{D}$ positions of the objects. The relative position determined using the $3 \mathrm{D}$ 
coordinate measuring machine was used as a ground truth.
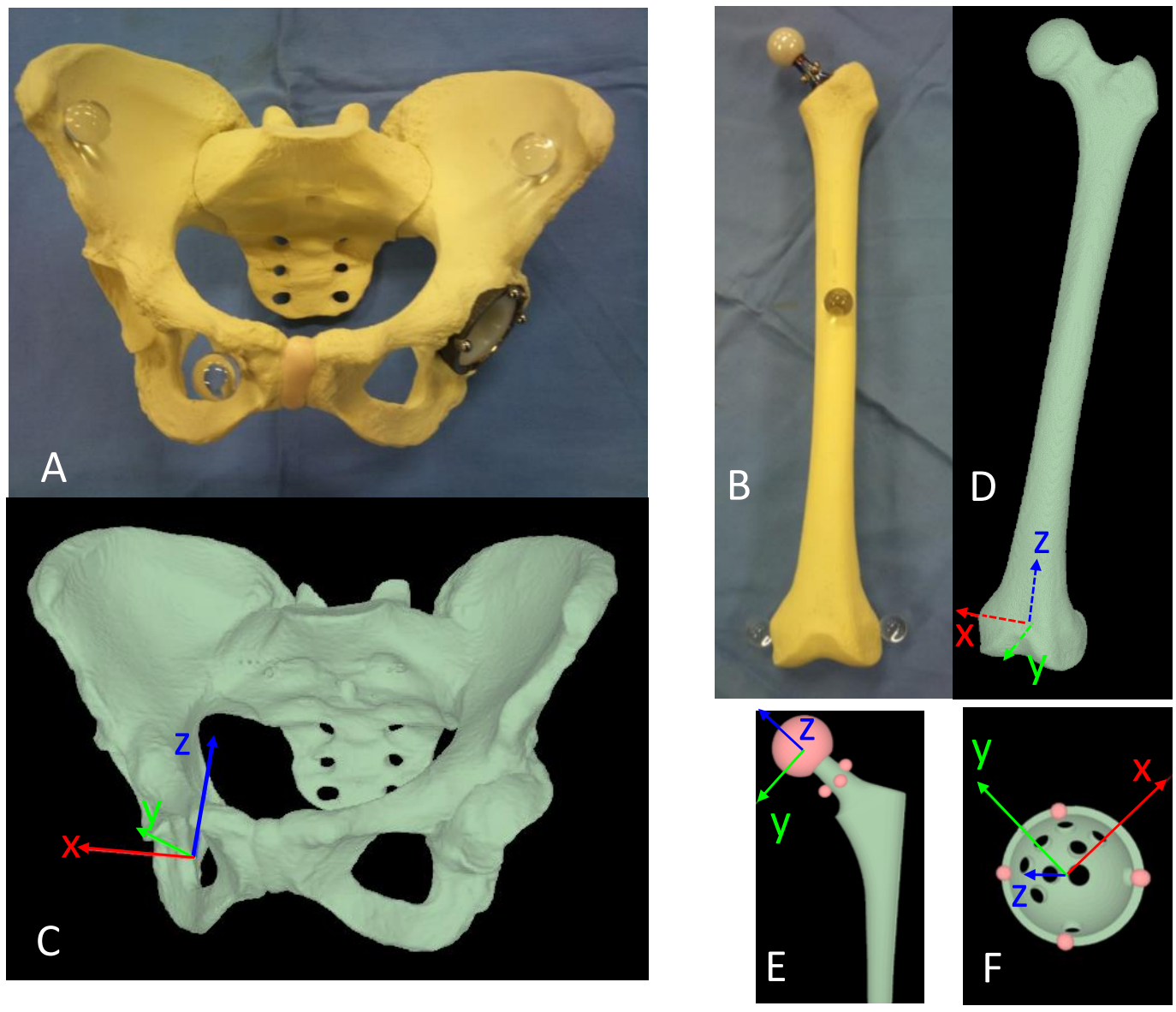

Fig. 1 Sawbone pelvis, femur, and THA implants with the corresponding 3D computer models. The sawbone pelvis (A) and femur (B) were implanted with the acetabular cup and femoral stem with the head on the left side. Local coordinate systems were determined in the pelvis model (C), femoral model (D), femoral stem CAD model (E), and acetabular cup CAD model (F) using the markers attached.

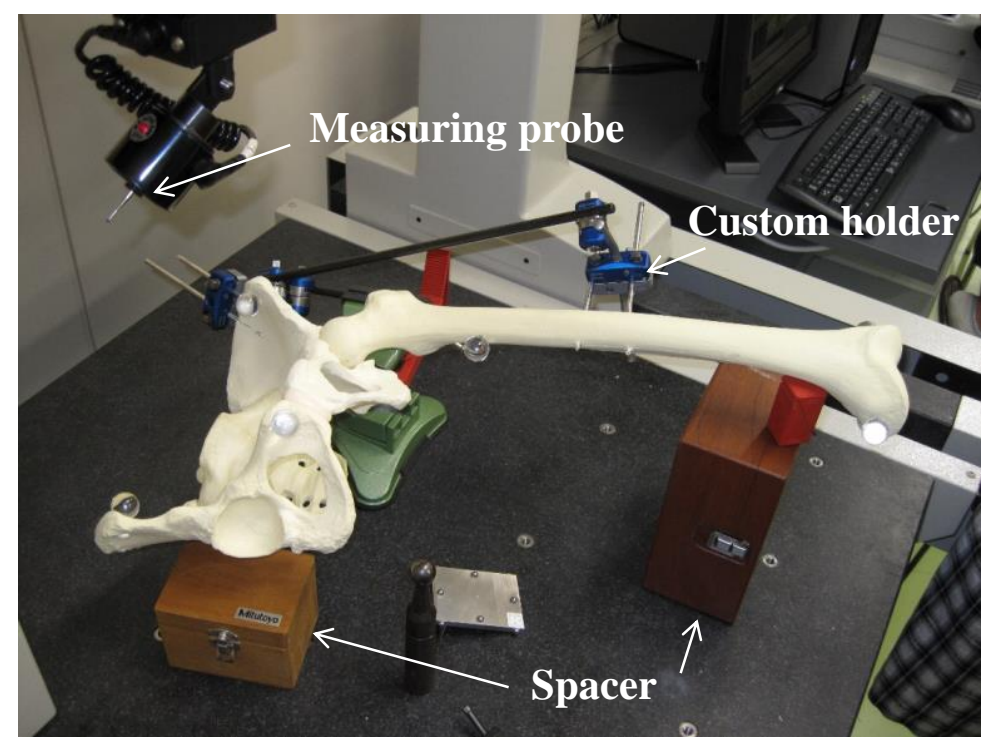

Fig. 2 Set-up for measuring relative position using the 3D-coordinate measuring machine. The sawbone femur and pelvis were fixed using a custom holder. 


\subsection{Image registration}

The fixed femur and pelvis were imaged at the three different positions using a biplanar X-ray system. This system consists of a rotation table positioned at $0^{\circ}$ and $60^{\circ}$ relative to the optical axis of a single X-ray source to capture images of the hip joint from frontal and oblique views with a distance of approximately $2.7 \mathrm{~m}$ from the X-ray source to the subject for each position. Two acrylic calibration panels with 40 spherical stainless markers for each were set on the table to cover the volume of the lower extremity (Fig. 3). The panels were imaged with the subject simultaneously. Two cassettes rotated with the table, while normal directions were set to align with the optical axis. Each cassette contained three 14" $\times 14$ " imaging plates that were to be digitized in 8-bit gray intensity with 0.2-mm resolution (FCR; Fuji, Tokyo, Japan).
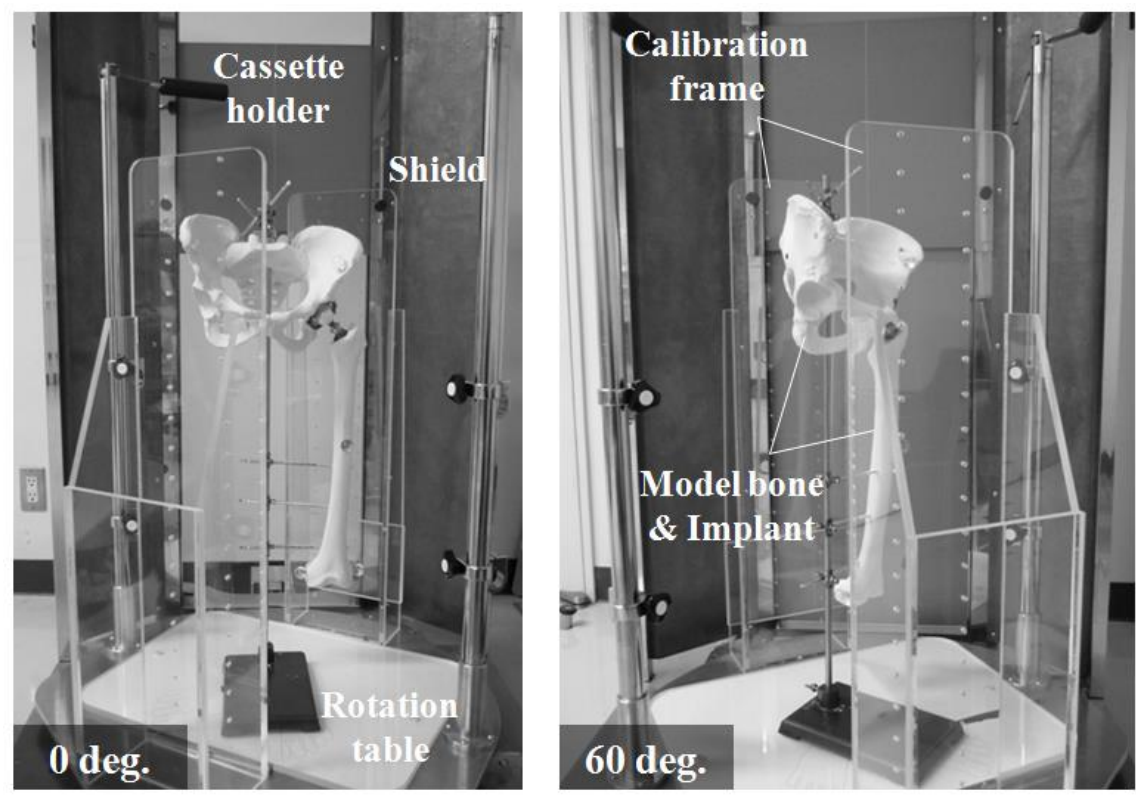

Fig. 3 Rotation table for bi-plane radiography. The rotation table was positioned at $0^{\circ}$ and $60^{\circ}$ relative to the optical axis of a single X-ray source.

For each table position, X-ray tube calibration was performed beforehand to determine the projection matrix (Faugeras, 1993). The projection matrix provides 3D positioning of the focus of the X-ray source, and the image plane with respect to the calibration frame, enabling projections of 3D objects to be replicated on the image plane following X-ray exposure (Fig. 4). Contour points of the femur, pelvis and implants in biplanar radiographs were detected using the method described by Canny (1986), and denoted as $\mathbf{p}_{i}(i=1, \ldots, N)$. Projected outline points of each 3D model were the finite edge points of the 2D shadow created from the projections of all visible triangular surfaces of the 3D model, and denoted as $\mathbf{q}_{j}(j=1, \ldots, M)$.

For the $i$-th point of the object contour, $\mathbf{p}_{i}$, the closest point was searched from all points of the projected outline of the corresponding model, $\mathbf{q}_{j}(j=1, \ldots, M)$, and denoted as $\mathbf{q}_{c}$. This searching process was repeated for all object contour points. The distance between $\mathbf{p}_{i}$ and $\mathbf{q}_{c}$ was summed over all object contour points and subsequently normalized by the total number of points, $N$. Object function $F$ represents the sum of normalized distances determined from frontal and oblique images:

$$
F=\sum_{i=1}^{N^{F R}}\left|\mathbf{p}_{i}^{F R}-\mathbf{q}_{c}^{F R}\right| / N^{F R}+\sum_{i=1}^{N^{O B}}\left|\mathbf{p}_{i}^{O B}-\mathbf{q}_{c}^{O B}\right| / N^{O B}
$$

where superscripts $F R$ and $O B$ denote the values standing for frontal and oblique images. The 3D position of each model (6-DOF parameters) was recovered by minimizing $F$ using the downhill simplex algorithm (Nelder and Mead, 1965). Ten sets of initial 6-DOF parameters for minimization were arbitrarily chosen from $\pm 5.0^{\circ}$ and $\pm 5.0 \mathrm{~mm}$ of true values of each relative position. The minimization procedure terminates if either the number of iterations exceeds 500 or the relative change in $F$ is below 0.00005 . 


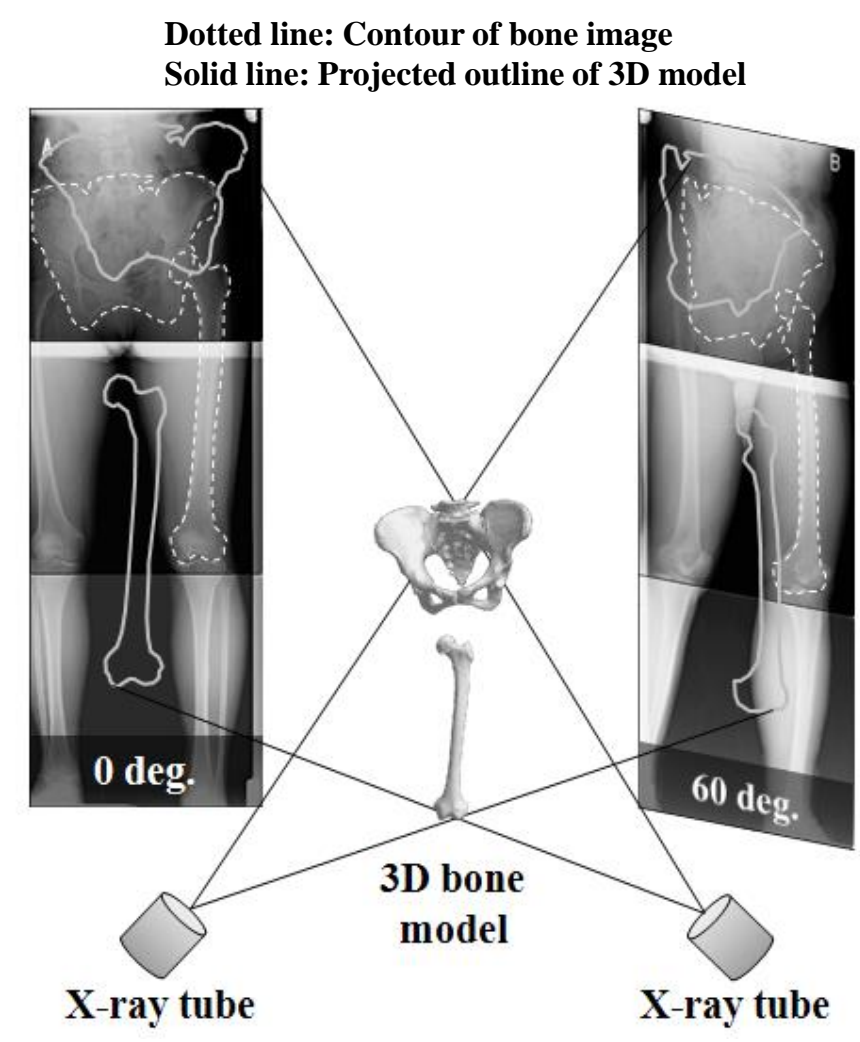

Fig. 4 Virtual biplanar X-ray imaging system. Projected outline points of each 3D model were the finite edge points of the $2 \mathrm{D}$ shadow. The averaged distance between the outline points and contour points of bone was minimized to recover the $3 \mathrm{D}$ position of bone.

\section{Results}

Mean values and standard deviations (SDs) of absolute errors in relative position parameters are shown in Table 1 for the three positions of femur and pelvis, femoral and acetabular components, femur and femoral component, and pelvis and acetabular component. Mean absolute errors were less than $1.27 \pm 0.79 \mathrm{~mm}$ and $0.99 \pm 0.52^{\circ}$ for femur and pelvis, $1.64 \pm 0.66 \mathrm{~mm}$ and $1.54 \pm 0.39^{\circ}$ for femoral and acetabular components, $1.58 \pm 0.29 \mathrm{~mm}$ and $1.75 \pm 0.69^{\circ}$ for femur and femoral component, and $1.51 \pm 0.49 \mathrm{~mm}$ and $1.24 \pm 0.14^{\circ}$ for pelvis and acetabular component.

Table 1. Mean and SD of absolute errors for estimating 6-DOF parameters of relative positions.

\begin{tabular}{|c|c|c|c|c|c|c|c|c|}
\hline & & & \multicolumn{3}{|c|}{ Translation, $\mathrm{mm}$} & \multicolumn{3}{|c|}{ Rotation, $^{\circ}$} \\
\hline & & & $\mathrm{x}$ & $\mathrm{y}$ & $\mathrm{Z}$ & $\mathrm{x}$ & $\mathrm{y}$ & $\mathrm{Z}$ \\
\hline \multirow{6}{*}{ Femur/pelvis } & \multirow{2}{*}{ Extension } & Mean & 0.65 & 1.26 & 0.79 & 0.97 & 0.41 & 0.96 \\
\hline & & SD & 0.63 & 0.94 & 0.36 & 0.25 & 0.11 & 0.44 \\
\hline & \multirow{2}{*}{$\begin{array}{l}\text { Anterior } \\
\text { tilt }\end{array}$} & Mean & 0.66 & 1.27 & 0.41 & 0.20 & 0.37 & 0.64 \\
\hline & & SD & 0.39 & 0.79 & 0.11 & 0.15 & 0.09 & 0.27 \\
\hline & \multirow{2}{*}{$\begin{array}{l}\text { Posterior } \\
\text { tilt }\end{array}$} & Mean & 0.79 & 1.08 & 0.86 & 0.27 & 0.25 & 0.99 \\
\hline & & SD & 0.51 & 0.82 & 0.33 & 0.09 & 0.10 & 0.52 \\
\hline \multirow{6}{*}{$\begin{array}{l}\text { Femoral/acetabular } \\
\text { components }\end{array}$} & \multirow{2}{*}{ Extension } & Mean & 0.98 & 0.44 & 0.22 & 0.37 & 0.74 & 0.26 \\
\hline & & SD & 0.13 & 0.18 & 0.10 & 0.07 & 0.47 & 0.17 \\
\hline & \multirow{2}{*}{$\begin{array}{l}\text { Anterior } \\
\text { tilt }\end{array}$} & Mean & 1.46 & 0.40 & 1.08 & 0.77 & 1.54 & 0.72 \\
\hline & & SD & 0.37 & 0.09 & 0.35 & 0.31 & 0.39 & 0.47 \\
\hline & \multirow{2}{*}{$\begin{array}{l}\text { Posterior } \\
\text { tilt }\end{array}$} & Mean & 1.64 & 1.46 & 0.28 & 1.08 & 0.84 & 0.22 \\
\hline & & SD & 0.66 & 0.33 & 0.22 & 0.59 & 0.84 & 0.22 \\
\hline
\end{tabular}


Table 1. Continued.

\begin{tabular}{|c|c|c|c|c|c|c|c|c|}
\hline & & & \multicolumn{3}{|c|}{ Translation, mm } & \multicolumn{3}{|c|}{ Rotation, $^{\circ}$} \\
\hline & & & $\mathrm{x}$ & $\mathrm{y}$ & $\mathrm{Z}$ & $\mathrm{x}$ & $\mathrm{y}$ & $\mathrm{Z}$ \\
\hline \multirow{6}{*}{$\begin{array}{l}\text { Femur/femoral } \\
\text { component }\end{array}$} & \multirow{2}{*}{ Extension } & Mean & 1.10 & 0.55 & 0.16 & 0.59 & 1.48 & 1.32 \\
\hline & & $\mathrm{SD}$ & 0.22 & 0.11 & 0.09 & 0.05 & 0.52 & 0.21 \\
\hline & \multirow{2}{*}{$\begin{array}{l}\text { Anterior } \\
\text { tilt }\end{array}$} & Mean & 0.23 & 1.58 & 1.04 & 0.39 & 1.75 & 0.59 \\
\hline & & $\mathrm{SD}$ & 0.16 & 0.29 & 0.13 & 0.15 & 0.69 & 0.34 \\
\hline & \multirow{2}{*}{$\begin{array}{l}\text { Posterior } \\
\text { tilt }\end{array}$} & Mean & 1.26 & 1.45 & 0.81 & 0.57 & 1.61 & 0.35 \\
\hline & & $\mathrm{SD}$ & 0.39 & 0.60 & 0.41 & 0.22 & 0.84 & 0.15 \\
\hline \multirow{6}{*}{$\begin{array}{l}\text { Pelvis/acetabular } \\
\text { component }\end{array}$} & \multirow{2}{*}{ Extension } & Mean & 0.41 & 0.76 & 0.43 & 0.65 & 1.24 & 1.10 \\
\hline & & SD & 0.13 & 0.13 & 0.12 & 0.33 & 0.14 & 0.41 \\
\hline & \multirow{2}{*}{$\begin{array}{l}\text { Anterior } \\
\text { tilt }\end{array}$} & Mean & 0.59 & 0.91 & 0.25 & 0.60 & 0.43 & 0.61 \\
\hline & & $\mathrm{SD}$ & 0.27 & 0.42 & 0.18 & 0.36 & 0.27 & 0.29 \\
\hline & \multirow{2}{*}{$\begin{array}{l}\text { Posterior } \\
\text { tilt }\end{array}$} & Mean & 1.13 & 1.51 & 1.21 & 0.52 & 0.56 & 0.67 \\
\hline & & $\mathrm{SD}$ & 0.23 & 0.49 & 0.39 & 0.38 & 0.32 & 0.48 \\
\hline
\end{tabular}

\section{Discussion}

This study validated the image registration technique for measuring the alignment of natural and implanted hip joints and component positions after THA in a standing posture. Overall, mean absolute errors were within $1.64 \mathrm{~mm}$ in translations and $1.75^{\circ}$ in rotations. Previous studies have reported indirect measurement of bony kinematics of the hip joint using skin marker-based motion analysis (Otake, et al., 2005). Although these indirect methods allow subjects to perform various functional activities, soft tissue artifacts would lower their accuracy and precision (Cappozzo, et al., 1996). A 2D/3D registration technique that combines X-ray imaging of hip joint and 3D geometrical models of implant and/or bone enables measurement of the relative position of the joint without the limitation of soft tissue artifacts. Previous studies have reported measurement of THA kinematics and cup orientation using single-plane fluoroscopy or radiography. Komistek et al. (2002) investigated in vivo hip separation after THA, but did not report the accuracy of translation along out-of-plane direction and rotations of the implants. Koyanagi et al. (2011) reported in vivo kinematic analysis of squatting after THA with maximum root mean square errors of $3.12 \mathrm{~mm}$ and $1.45^{\circ}$ in translations and rotations. Penny et al. (2007) determined cup orientation in THA within mean absolute errors of $1.4 \mathrm{~mm}$ or $1.4^{\circ}$. However, these investigations were limited to implanted joints.

Using a dual fluoroscopic system, accuracy and precision were reported as $0.33 \pm 0.81 \mathrm{~mm}$ in translation and $0.45 \pm$ $0.65^{\circ}$ in rotation for measuring THA motion (Tsai, et al., 2013), $0.2 \pm 0.3 \mathrm{~mm}$ and $0.2 \pm 0.8^{\circ}$ for translation and rotation during functional activities of natural hips (Martin, et al., 2011), and $0.93 \pm 1.13 \mathrm{~mm}$ in translation and $0.59 \pm 0.82^{\circ}$ in rotation for measuring natural hip kinematics (Lin, et al., 2013), where radio-stereometric analysis was used to obtain a ground truth.

The present study demonstrated that the 3D LAAS offered similar or better accuracy than the single-plane imaging technique, but less accuracy than the dual fluoroscopic system. On the other hand, clinical validations of a CT-based navigation system have reported mean errors within $1.8 \pm 1.6^{\circ}$ for cup angle and $1.9 \pm 1.5 \mathrm{~mm}$ for cup position (Iwana, et al., 2013), and $0.6 \pm 4.8^{\circ}$ for stem anteversion and $-0.2 \pm 1.8^{\circ}$ for stem valgus angle (Kitada, et al., 2011). As these results were comparable to ours, 3D LAAS appears applicable to evaluate 3D alignment and implant position of the hip joint. Moreover, the 3D LAAS can capture the entire lower extremity. This offers an advantage over dual fluoroscopic systems, which only capture images of a single joint, not the entire lower limb.

The use of CT may lower the applicability of our method, due to concerns about exposure to radiation. However, the effective radiation dose of $6 \mathrm{mSv}$ from CT to the hip is comparable to the annual effective dose from natural background radiation of about $3 \mathrm{mSv}$ (Mettler, et al., 2008).

Several limitations to the present study must be considered. The results of the present study were obtained from experiments on sawbone specimens. This eliminated the soft tissues surrounding the hip joint that may reduce the quality of X-ray image and the reliability of contour extraction from bone. Studies examining actual patients are thus needed to confirm the clinical applicability of this procedure. We analyzed only one relative position between the implant and target 
bone. Further investigations with various implant positions simulating malposition are warranted.

\section{Conclusions}

The present study validated the 3D LAAS for measurement of the alignment of natural and implanted hip joints and component positions after THA in a standing posture. Overall, mean absolute errors were within $1.64 \mathrm{~mm}$ in translations and $1.75^{\circ}$ in rotations, demonstrating that the 3D LAAS is a useful tool for quantifying 3D alignment of the hip joint and implant position in a standing position.

\section{Acknowledgements}

This research was supported by JSPS KAKENHI Grant Number 24246025, Grant-in-Aid for Scientific Research (A).

\section{References}

Ariumi A., Sato T., Kobayashi K., Koga Y., Omori G., Minato I., Endo N., Three-dimensional lower extremity alignment in the weight-bearing standing position in healthy elderly subjects, Journal of Orthopaedic Science, Vol.15 (2010), pp.64-70.

Canny A., A computational approach to edge detection, IEEE Transactions on Pattern Analysis and Machine Intelligence, Vol.8, No.6 (1986), pp.679-698.

Cappozzo A., Catani F., Leardini A., Benedetti M.G., Della Croce U., Position and orientation in space of bones during movement : experimental artifacts, Clinical Biomechanics, Vol.11, No.2 (1996), pp.90-100.

D'Lima D.D., Urquhart A.G., Buehler K.O., Walker R.H., Colwell C.W., The Effect of the orientation of the acetabular and femoral components on the range of motion of the hip at different head-neck ratios, Journal of Bone and Joint Surgery, Vol.82-A, No.3 (2000), pp.315-321.

Faugeras O., Three-Dimensional Computer Vision (1993), pp.33-68, MIT Press.

Iwana D., Nakamura N., Miki H., Kitada M., Hananouchi T., Sugano N., Accuracy of angle and position of the cup using computed tomography-based navigation systems in total hip arthroplasty, Computer Aided Surgery, Vol.18, No.5-6 (2013), 187-194.

Jolles B.M., Zangger P., Leyvraz P.-F., Factors predisposing to dislocation after primary total hip arthroplasty, Journal of Arthroplasty, Vol.17, No.3 (2002), pp.282-288.

Jolles B.M., Genoud P., Hoffmeyer P., Computer-assisted cup placement techniques in total hip arthroplasty improve accuracy of placement, Clinical Orthopaedics and Related Research, No.426 (2004), pp.174-179.

Kesteris U., Hardinge K., Ilchmann T., Wingstrand H., Polyethylene wear in prosthetic hips with loose components, Journal of Arthroplasty, Vol.18, No.1 (2003), pp.10-15.

Kitada M., Nakamura N., Iwana D., Kakimoto A., Nishii T., Sugano N., Evaluation of the accuracy of computed tomography-based navigation for femoral stem orientation and leg length discrepancy, Journal of Arthroplasty, Vol.26, No.5 (2011), pp.674-679.

Kobayashi K., Sakamoto M., Tanabe Y., Ariumi A., Sato T., Omori G., Koga Y., Automated image registration for assessing three-dimensional alignment of entire lower extremity and implant position using bi-plane radiography. Journal of Biomechanics, Vol.42 (2009), pp.2818-2822.

Komistek R.D., Dennis D.A., Ochoa J.A., Haas B.D., Hammill C., In vivo comparison of hip separation after metal-onmetal or metal-on-polyethylene total hip arthroplasty, Journal of Bone and Joint Surgery, Vol.84-A (2002), pp.18361841.

Koyanagi K., Sakai S., Yamazaki T., Watanabe T., Akiyama K., Sugano N., Yoshikawa Y., Sugamoto K., In vivo kinematic analysis of squatting after total hip arthroplasty, Clinical Biomechanics, Vol.26 (2011), pp.477-483.

Lin H., Wang S., Tsai T.-Y., Li G., Kwon Y.-M., In-vitro validation of a non-invasive dual fluoroscopic imaging technique for measurement of the hip kinematics, Medical Engineering \& Physics, Vol.35 (2013), pp.411-416.

Malik A., Maheshwari A., Dorr L.D., Impingement with total hip replacement, Journal of Bone and Joint Surgery, Vol.89A, No.8 (2007), pp.1832-1842.

Martin D.E., Greco N.J., Klatt B.A., Wright V.J., Anderst W.J., Tashman S., Model-based tracking of the hip: implications 
for novel analyses of hip pathology, Journal of Arthroplasty, Vol.26, No.1 (2011), pp.88-97.

Mellon S.J., Kwon Y.-M., Glyn-Jones S., Murray D.W., Gill H.S., The effect of motion patterns on edge-loading of metalon-metal hip resurfacing, Medical Engineering \& Physics, Vol.33 (2011), pp.1212-1200.

Mettler F.A. Jr., Huda W., Yoshizumi T.T., Mahesh M., Effective doses in radiology and diagnostic nuclear medicine: A catalog, Radiology, Vol.248, No.1 (2008), pp.254-263.

Nelder J.A. and Mead R., A simplex method for function minimization, Computer Journal, Vol.7 (1965), pp.308-13.

Otake Y., Suzuki N., Hattori A., Hagio K., Sugano N., Yonenobu K., Ochi T., Four-dimensional model of the lower extremity after total hip arthroplasty, Journal of Biomechanics, Vol.38, No.12 (2005), pp.2397-2405.

Penney G.P., Edwards P.J., Hipwell J.H., Slomczykowski M., Revie I., Hawkes D.J., Postoperative calculation of acetabular cup position using 2-D-3-D registration, IEEE Transactions on Biomedical Engineering, Vol.54, No.7 (2007), pp.1342-1348.

Sasagawa K., Watanabe S., Kobayashi K., Sakamoto M., Tanabe Y., Sato T., Koga Y., Accuracy examination of threedimensional bone surface model using MRI and CT. Japanese Journal of Clinical Biomechanics, Vol.29 (2008), pp.397-402 (in Japanese).

Sato T., Koga Y., Omori G., Three-dimensional lower extremity alignment assessment system. Application to evaluation of component position after total knee arthroplasty, Journal of Arthroplasty, Vol.19, No.5 (2004), pp.620-628.

Sato T., Koga Y., Sobue T., Omori G., Tanabe Y., Sakamoto M., Quantitative 3-dimensional analysis of preoperative and postoperative joint lines in total knee arthroplasty, Journal of Arthroplasty, Vol.22, No.4 (2007), pp.560-568.

Tsai T.-Y., Li J.-S., Wang S., Lin H., Malchau H., Li G., Rubash H., Kwon Y.-M., A novel dual fluoroscopic imaging method for determination of THA kinematics: In-vitro and in-vivo study, Journal of Biomechanics, Vol.46 (2013), pp.1300-1304.

Yoshimine F., The safe-zones for combined cup and neck anteversions that fulfill the essential range of motion and their optimum combination in total hip replacements, Journal of Biomechanics, Vol.39 (2006), pp.1315-1323. 